\title{
Reviewer Acknowledgments 2020—Addendum
}

Published online: 19 October 2020

(C) Springer Science+Business Media, LLC, part of Springer Nature 2020

Due to a clerical error, several reviewers were mistakenly excluded from our previous list of "Reviewer Acknowledgments". Our apologies for any confusion this error may have caused.

In addition to the previous "Reviewer Acknowledgments" list of reviewers, we also acknowledge the following 62 reviewers for their contributions to Obesity Surgery. Accordingly, the maximum number of individual reviewer contributions in the 12-month period between 1 September 2019 and 31 August 2020 should be indicated as 17 (rather than 6). Thank you all!

\author{
Hussam Zuhir Adi \\ Md Tanveer Adil \\ Raad Al Mehdi \\ Luciano Antozzi \\ Alexander Ayzengart \\ Ambar Banerjee \\ Kumar Belani \\ Mohit Bhandari \\ Laurent Biertho \\ Esther Maria Bonrath \\ Lauren E Bradley \\ James Byrne \\ Enrico Camporesi \\ William Reginald Joseph Carr \\ Carlos Alberto Casalnuovo \\ Marco Castagneto \\ Chong-Chi Chiu \\ Elie K Chouillard \\ Eva M Conceição \\ Catalin Copaescu \\ Andrew Currie \\ Greg Dakin \\ Adrian George Dan \\ Anne Pugel Ehlers \\ Marwan El Ghoch \\ Paul Enochs \\ Alex Escalona
}

\author{
Angela Fitch \\ Chandra Hassan \\ Abdulzahra Hussain \\ Valentina Ivezaj \\ Ignace Maria Christianus Janssen \\ Maria Jose Leiva \\ Leon Katz \\ John Kelly \\ Mohammad Kermansaravi \\ Guowei Kim \\ Alexander Klaus \\ Daisuke Kobayashi \\ Panagiotis Lainas \\ Andriy Lavryk \\ Andrea Lazzati \\ Ryan Lehmann \\ Luc Lemmens \\ Jose Antonio Lopez Martinez \\ Raymond McHenry \\ Ian Michell \\ Ruairi Moulding \\ Fady Moustarah \\ Philipp Nett \\ Athanasios Pantelis \\ Zvi Howard Perry \\ Arun Prasad \\ Kevin Reavis \\ Keith Scharf \\ Colleen Schreyer \\ Shahab Shahabi Shahmiri \\ Cassie I Story \\ Michael L Talbot \\ Surendra Ugale \\ Joseph Vitello \\ Kurt Widhalm
}

Publisher's Note Springer Nature remains neutral with regard to jurisdictional claims in published maps and institutional affiliations. 